\title{
Cardiac pharmacologic stress: does the gender matters?
}

\author{
Claudio Tinoco Mesquita ${ }^{1,2} \cdot$ Wilter dos Santos $\operatorname{Ker}^{1,2}$
}

Published online: 14 September 2019

(C) Springer-Verlag GmbH Germany, part of Springer Nature 2019

Men and women have differences and similarities. Some of them are easy to determine and to understand. Some are subtler and more difficult to evaluate. The comprehension of these characteristics is very important to better address health care issues. Cardiovascular disease (CVD) is the leading cause of mortality for women globally, accounting for up to one-third of all deaths in women worldwide [1]. Unfortunately, CVD remains understudied, underdiagnosed, and undertreated in women [2]. There are two main aspects to address in this topic: sex differences resulting from biological factors and gender differences related to social, environmental, and community factors [2]. Women present with angina as the first manifestation of coronary artery disease (CAD) more frequently than men. Women have more diverse symptom presentation than do men: with pain or discomfort in jaw, neck, and interscapular area; associated epigastric discomfort and associated nausea, dyspnea, and fatigue [3]. Acute myocardial infarction (AMI) without chest pain is more common in women and the mortality rate is higher than men [3]. Myocardial ischemia in women is more commonly associated with nonobstructive disease of the epicardial coronary arteries, microvascular disease, and coronary vasospasm. This different pathophysiology is not benign. The WISE Study demonstrated an elevated risk of all-cause mortality among women with signs and symptoms of ischemia but without obstructive coronary artery disease $(13 \%)$ as compared with women of

This article is part of the Topical Collection on Editorial

Claudio Tinoco Mesquita

claudiotinocomesquita@id.uff.br

1 Radiology Department (Ebserh/UFF), Hospital Universitário Antonio Pedro, Niteroi, Rio de Janeiro, Brazil

2 Hospital Pró-Cardíaco, Rio de Janeiro, Brazil approximately the same age during the same period (2.8\%) [4]. Women are less frequently referred for appropriate treatment during an AMI compared with men despite proven mortality benefits of therapy [2]. Figure 1 provides some of the most important characteristics that the cardiologist and nuclear physician must address in the management of women with suspected or know CAD.

We have to congratulate Katsikis et al. for the well-designed paper published in European Journal of Nuclear Medicine and Molecular Imaging that demonstrated that women exhibit a higher rate of any side-effect and an increased HRR after regadenoson administration compared with their male counterparts [5]. The authors found several differences in adverse effects of regadenoson: women had a significantly higher rate of chest pain, gastrointestinal discomfort, dizziness, and headache. Besides that, women demonstrated a higher median HR-response compared with men [5]. Gender differences with pharmacologic stress were first described by Thomas et al. with adenosine. These authors found that women were more likely to experience adverse reactions to adenosine during myocardial perfusion imaging than men [6]. One potential explanation for the increase in adverse events in women is that generally greater percent body fat in women may result in a relatively higher plasma concentration of adenosine because of a smaller volume of distribution in women. Similar mechanism can contribute to the differential effects in women observed in this study [5]. The professional responsible for the cardiac stress must be alert to the potential increased value of aminophylline use and concomitant low-level exercise to reduce the adverse effects of regadenoson infusion in women [5].

The HR-response (percentage change from baseline) to regadenoson during stress is a nonperfusional relevant prognostic factor. HR-response $<30 \%$ is associated with an increased risk of death 


\section{Women versus Men in Coronary Artery Disease}
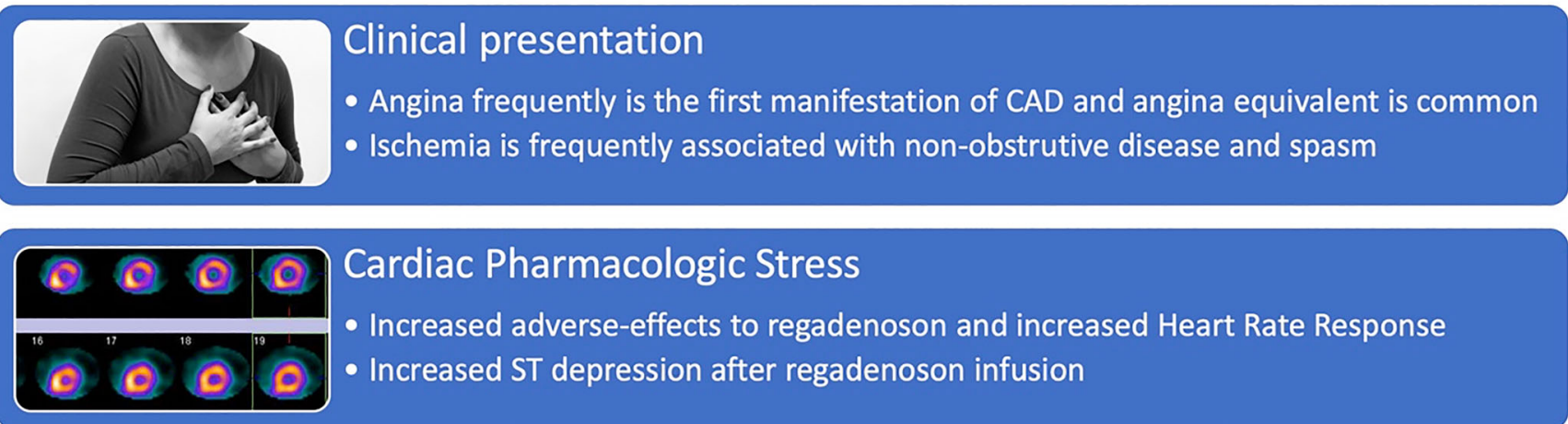

Cardiac Pharmacologic Stress

Increased adverse-effects to regadenoson and increased Heart Rate Response

- Increased ST depression after regadenoson infusion

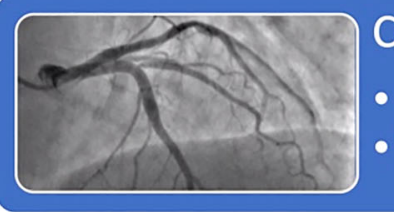

Coronary Anatomy

- Increased prevalence of non-obstructive disease with adverse prognosis

- Decreased use of resources to treat acute myocardial infarction

Fig. 1 Selected women characteristics in coronary artery disease that are relevant to the cardiologist and to the imaging physician

[7]. In the study of Katsikis et al., women had an increased HRR after regadenoson administration compared with men. The implications of these findings are currently not understood. Gender-related differences in the function of the autonomic nervous system and hormonal activity can explain part of these findings [5].

Gender-related differences with the use of regadenoson can be found in the electrocardiographic response [6]. ST depression after regadenoson infusion is more frequently encountered in women in 3:1 ratio and is associated with independent prognostic value beyond clinical and perfusional data [8]. One limitation of the Katsikis study is that it did not evaluate the electrocardiographic response, and the results of this analysis could be compared to other clinical and hemodynamic behaviors. Another limitation was the use of propensity score method that is commonly employed in an attempt to reduce bias from concomitant confounding variables (to correct for many baseline imbalances). Although this technique can balance observed baseline covariates between groups, it cannot balance unmeasured characteristics and confounders [9]. The definitive answer about the intensity of gender-differences in regadenoson should be derived from a randomized trial but is very improbable that a study like that changes significantly our knowledge about this topic.

Katsikis et al. should be congratulated because they are contributing to the closing of the gap in sex disparities [5]. One of the priorities to improve outcomes in women with CHD is to increase the awareness of healthcare providers of sex-specific symptoms and clinical presentation in CHD [2]. Gender matters including during pharmacologic cardiac stress.

\section{Compliance with ethical standards}

Conflict of interest The authors declare that they have no conflict of interest.

Images are from the corresponding author's personal archive.

\section{References}

1. Gholizadeh L, Davidson P. More similarities than differences: an international comparison of CVD mortality and risk factors in women. Health Care Women Int. 2008;29(1):3-22.

2. Mehta LS, Beckie TM, DeVon HA, Grines CL, Krumholz HM, Johnson MN, Lindley KJ, Vaccarino V, Wang TY, Watson KE, Wenger NK; American Heart Association Cardiovascular Disease in Women and Special Populations Committee of the Council on Clinical Cardiology, Council on Epidemiology and Prevention, Council on Cardiovascular and Stroke Nursing, and Council on Quality of Care and Outcomes Research. Acute myocardial infarction in women: a scientific statement from the American Heart Association. Circulation. 2016;133(9):916-47.

3. Wenger NK. Clinical presentation of CAD and myocardial ischemia in women. J Nucl Cardiol. 2016;23(5):976-85.

4. Kenkre TS, Malhotra P, Johnson BD, et al. Ten-year mortality in the WISE Study (Women's Ischemia Syndrome Evaluation). Circ Cardiovasc Qual Outcomes. 2017;10(12):1-9.

5. Katsikis A, Kyrozi E, Manira V, Theodorakos A, Malamitsi J, Tsapaki V, Iakovou I, Voudris V, Kolovou G, Koutelou M. Genderrelated differences in side-effects and hemodynamic response to 
regadenoson in patients undergoing SPECT myocardial perfusion imaging. Eur J Nucl Med Mol Imaging. 2019. https://doi.org/10. 1007/s00259-019-04463-9.

6. Thomas GS, Prill NV, Majmundar H, et al. Treadmill exercise during adenosine infusion is safe, results in fewer adverse reactions, and improves myocardial perfusion image quality. J Nucl Cardiol. 2000;7(5):439-46.

7. Andrikopoulou E, AlJaroudi WA, Farag A, et al. The reproducibility and prognostic value of serial measurements of heart rate response to regadenoson during myocardial perfusion imaging. Eur J Nucl Med Mol Imaging. 2016;43(8):1493-502. https://doi.org/10.1007/ s00259-016-3380-y.
8. Doukky R, Nigatu A, Khan R, et al. Prognostic significance of ischemic electrocardiographic changes with regadenoson stress myocardial perfusion imaging. J Nucl Cardiol. 2018. https://doi.org/10. 1007/s12350-018-1415-4.

9. Reiffel JA. Propensity-score matching: optimal, adequate, or incomplete? J Atr Fibrillation. 2018;11(4):2018-9.

Publisher's note Springer Nature remains neutral with regard to jurisdictional claims in published maps and institutional affiliations. 